\title{
Potential of Supercapacitors in Novel Power Converters as Semi-ideal Lossless Voltage Droppers
}

\author{
Thilanga Ariyarathna, Dilini Jayananda, Nihal Kularatna and D. Alistair Steyn-Ross \\ School of Engineering \\ University of Waikato \\ Hamilton 3240, New Zealand \\ Email: taa21@students.waikato.ac.nz, dukju1@students.waikato.ac.nz,nihalkul@waikato.ac.nz, asr@waikato.ac.nz
}

\begin{abstract}
Electrical physics text book theory tells us that charging a capacitor is much less efficient than replenishing the energy in a discharged electro-chemical battery. If a fully discharged capacitor is pumped with a charge of $Q$ coulombs, it stores $\frac{1}{2} Q V^{2}$ while dissipating the same amount of energy in the loop resistance. However, if the same charge is pumped into a re-chargeable electrochemical cell of voltage $V$ the energy stored in the cell is $Q V$, while the wasted energy is determined by the loop resistance and the voltage difference across the resistance. If a rechargeable battery pack is to be replaced by a supercapacitor module, this difference could seriously affect the design of power converters required, since the power converter should stop charging at a certain point to avoid overcharging the capacitor bank. However, if a useful resistive load such as heater, DC-DC converter, inverter or a lamp load is used as a part of the loop resistance in a capacitor charging loop, a significant part of this loss can be recovered. One example of this is in the supercapacitor assisted low drop-out regulator (SCALDO) technique. This paper will detail the concept of circumvention of RC loop charging loss, theoretically quantifying the same in a generalized circuit, demonstrating how this can be applied in completely novel circuit topologies such as the supercapacitor assisted LED (SCALED) converter. The paper will provide experimental results of selected SCALDO implementations and early results of SCALED technique to support this theory.
\end{abstract}

\section{INTRODUCTION}

There are three common DC-DC converter design approaches in practical electronic systems: (i) linear regulators, (ii) switched-mode converters with magnetic components (inductors), and (iii) switched-mode converters without magnetic components (switched-capacitor converters) [1]. Linear regulators are ideal for applications that require extremely low noise such as audio amplifiers and low power consumer applications such as alarm panels [2]. In linear regulators, since there is a series power semiconductor to take up the difference between $V_{\text {reg }}$ and $V_{\text {in }}$, the efficiency is dependent on this difference. Typical efficiency of linear regulators is given by the ratio, $\eta=\frac{V_{\text {reg }}}{V_{\text {in }}}$ assuming that the control circuit consumes negligible power.

Inductor-based switch mode converters provide efficient and compact DC-DC converters. However they introduce RFI/ EMI issues in to the power supply [2-4]. The switched capacitor design (charge pump) uses capacitors only; inductors or transformers are not required. Compared to other converter types, charge pumps offer superior performance in applications which require low-noise operations. These converters have limited current capacity and require post regulators [5-8].

Low drop out (LDO) regulators deliver all of the advantages of linear regulators with an acceptably high efficiency. An LDO differs from a standard linear regulator because of its capability of operating with a very small voltage difference between the regulated output voltage level and the unregulated input voltage. Typically an LDO can offer a drop-out (difference between input and output voltage) of less than 0.3 $\mathrm{V}$ while a standard linear voltage regulator offers a drop-out voltage of $1 \mathrm{~V}$ or more $[9,10]$. This makes LDOs ideal for battery-powered portable devices. The restricted input voltage range is the main limitation associated with LDOs.

Electrical double-layer capacitors, commonly known as supercapacitors or ultracapacitors, have typical capacitance values of order one million times larger than standard capacitors used in electronic circuits. Supercapacitors have higher power density than rechargeable batteries and higher energy density than electrolytic capacitors. Applications of SCs include (i) memory backup, (ii) short-term power for Global Position System for mobile communication bursts and highbrightness flash in cell phones, (iii) hybrid batterySC systems for UPSs and power-quality enhancers, (iv) power tools, (v) integrated starter generator application systems in automotives. Their energy storage capabilities similar to rechargeable batteries [11-13].

Section II of the paper presents a mathematical analysis of the RC charging circuit generalized to cover SC applications. When applied to SC-based circuits, this analysis provides the theoretical foundation for a set of novel supercapacitor assisted (SCA) converter topologies developed at the Uni of Waikato:

- SCALDO: low dropout regulator

- SCASA: surge absorber

- SCATMA: temperature modification apparatus

- SCAHDI: high density inverter

- SCALED: LED

Sections III and IV demonstrate application of this theory to SCALDO and SCALED circuit topologies. SCALDO is proposed as a new and disruptive DC-DC converter topology [14] which permits the design of DC power supplies with very high ETEE (end-to-end efficiency) that are free of RFI/ EMI due to low cycling frequency. SCALED is aimed at DC 
microgrid applications. SCAHDI, SCATMA, SCASA are nontraditional examples for supercapacitor assisted technologies.

\section{Charging Behaviour of A Capacitor}

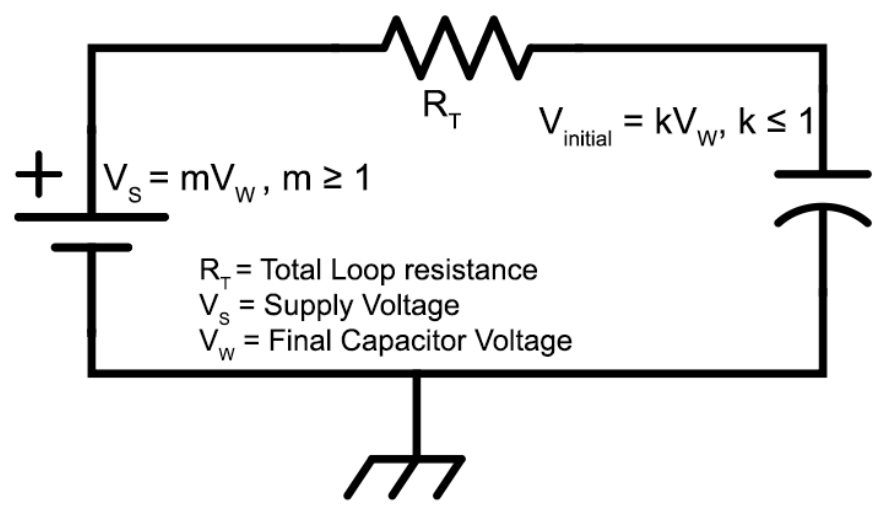

(a)

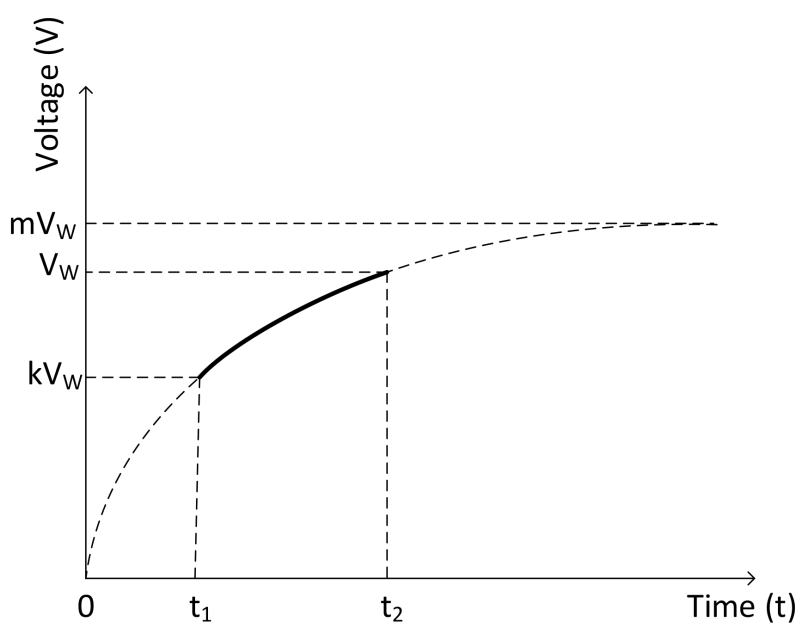

(b)

Fig. 1: (a) Capacitor charging circuit (b) Capacitor charging curve. We focus on the energy efficiency for partial charging from $k V_{\mathrm{W}}$ to $V_{\mathrm{W}}$ (bold curve)

An uncharged capacitor starting from a zero-charge condition achieves an energy content of $\frac{1}{2} C V^{2}$ after about five time constants, if the input source voltage is $V$. During this time, the resistive components in the loop waste an equal amount of energy, so the charging process is $50 \%$ efficient.

Here we extend this theory for the generalized case of Figure 1, where capacitor bank can be based on SCs, with a large circuit time constant, and the charging source voltage is larger than the rated voltage of the SC bank. If we charge a fully-discharged capacitor bank from 0 to $V_{\mathrm{W}}$ from a DC supply of $m V_{\mathrm{W}}$, the capacitor voltage at time $t$ is

$$
v(t)=m V_{\mathrm{W}}\left(1-e^{-t / R C}\right)
$$

following the curve in Figure 1b. Let $t_{1}$ and $t_{2}$ be the times required for $v(t)$ to reach $k V_{\mathrm{W}}(0<k \leq 1)$ and
$V_{\mathrm{W}}$ respectively. We seek to determine how the efficiency of the charging process varies as a function of $k$ (the initial voltage fraction) and $m$ (the power supply over-voltage factor). We take the generalized case of this over-rated DC source, since in large time constant based SC circuits we can use voltage detection circuits to disconnect the loop to prevent over-charging of the SC.

\section{A. Analysis of Charging Efficiency}

Charging an empty capacitor from a supply of voltage $m V_{\mathrm{W}}$ $(m \geq 1)$, the times taken to reach target voltage $k V_{\mathrm{W}}$ and $V_{\mathrm{W}}$ are given respectively by the expressions:

$$
\begin{aligned}
e^{-t_{1} / R C}= & \frac{m-k}{m} \text { and } e^{-t_{2} / R C}=\frac{m-1}{m} \\
m V_{\mathrm{W}}-V_{\mathrm{C}} & =m V_{\mathrm{W}}-m V_{\mathrm{W}}\left(1-e^{-t / R C}\right) \\
& =m V_{\mathrm{W}} e^{-t / R C}
\end{aligned}
$$

The increment in stored energy between time $t_{1}$ and $t_{2}$ is

$$
E_{\mathrm{C}}\left(t_{2}, t_{1}\right)=\frac{1}{2} C V_{\mathrm{W}}^{2}-\frac{1}{2} C\left(k V_{\mathrm{W}}\right)^{2}=\frac{1}{2} C V_{\mathrm{W}}^{2}\left(1-k^{2}\right)
$$

while the energy loss in the resistor over this time interval is given by the integral

$$
E_{\mathrm{R}}\left(t_{2}, t_{1}\right)=\int_{t_{1}}^{t_{2}} i^{2} R d t
$$

with

$$
i^{2}(t) R=\left(m V_{\mathrm{W}}-v(t)\right)^{2} / R=\frac{m^{2} V_{\mathrm{W}}^{2}}{R} e^{-2 t / R C}
$$

Integrating (4), and substituting end-point expressions (2), leads to

$$
E_{\mathrm{R}}\left(t_{2}, t_{1}\right)=\frac{1}{2} C V_{\mathrm{W}}^{2}\left(k^{2}-2 m k+2 m-1\right)
$$

giving an energy efficiency ratio

$$
\eta=\frac{E_{\mathrm{C}}}{E_{\mathrm{C}}+E_{\mathrm{R}}}=\frac{1-k^{2}}{2 m(1+k)}=\frac{1+k}{2 m}
$$

In the limit $k \rightarrow 0$ (no capacitor precharge) with $m \rightarrow$ 1 (no power supply over voltage factor) efficiency $\eta \rightarrow \frac{1}{2}$, recovering the standard textbook result. Equation (5) tells us that efficiency is enhanced if the capacitor carries a percentage (i.e., is never allowed to fully discharge during a cycle), thus avoiding the large energy losses that accrue when current is high.

if our goal is to minimize charging time, then we would set an over voltage factors $m>1$, but this implies higher current and reduced efficiency since $\eta \sim \frac{1}{m}$. These $(k, m)$ energy trends are visualised in Figure 2b, bottom layer: optimum efficiency is achieved when $(k, m) \rightarrow(1,1)$. 


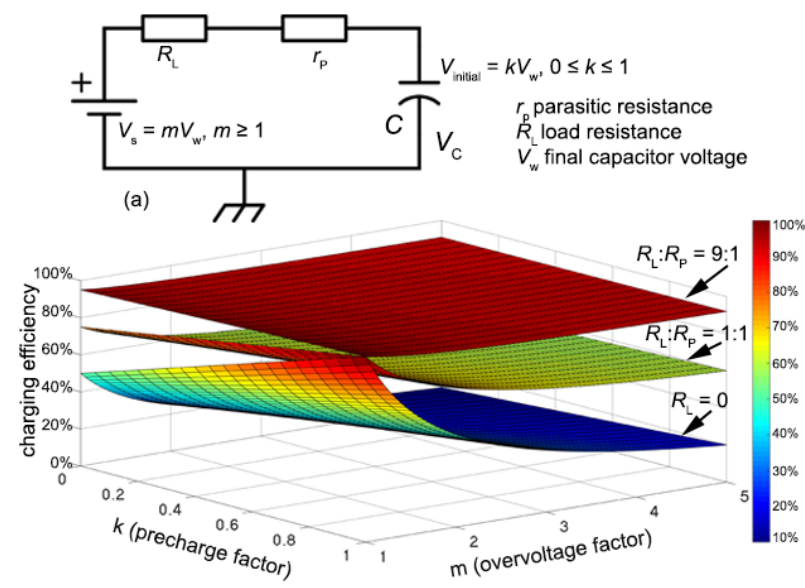

(b)

Fig. 2: Insertion of a useful resistance into the $\mathrm{RC}$ charging loop (a) modified circuit with useful resistance $R_{\mathrm{L}}$ inserted (b) Graphical representation of the circuit behaviour in terms of efficiency versus $m$ and $k$

Paradoxically, we can improve overall efficiency by inserting additional resistance $R_{\mathrm{L}}$ in to the charging loop, provided the new resistance is a heater, a loaded DC-DC converter, a loaded inverter or any other "useful" resistive load. This is because energy consumed by $R_{\mathrm{L}}$ does useful work before being dissipated as heat, while energy consumed by parasitic circuit resistance $r_{\mathrm{p}}$ is lost immediately. This allows us to refine the efficiency expression of (5) to read

$$
\eta=\frac{E_{\mathrm{C}}+E_{R_{\mathrm{L}}}}{E_{\mathrm{C}}+E_{R_{\mathrm{L}}}+E_{r_{\mathrm{P}}}}
$$

If we set $R_{\mathrm{L}} / r_{\mathrm{P}}=P>1$, then we obtain a performance boost

$$
\eta=\frac{1}{P+1}\left(P+\frac{1+k}{2 m}\right)
$$

which collapses to ratio (5) when $P \rightarrow 0$. For $R_{\mathrm{L}}: r_{\mathrm{p}}=1: 1$ and $9: 1$ respectively we obtain

$$
\eta(P=1)=\frac{1}{2}+\frac{1+k}{4 m} ; \eta(P=9)=\frac{9}{10}+\frac{1+k}{20 m}
$$

These trends are illustrated in Figure 2 middle and upper meshes.

\section{SUPERCAPACitor AS A LOSSLESS DROPPER}

In typical high frequency converters, inductors and capacitors serve as ideal energy storage devices. To store 100 $\mathrm{J}$ in an inductor carrying a $2 \mathrm{~A}$ current, based on $\frac{1}{2} L i^{2}$, we require an inductor of value $50 \mathrm{H}$, a bulkey component that is much too large for a compact DC-DC converter. In contrast, a standard $61 \mathrm{~cm}^{3}$ supercapacitor of $100 \mathrm{~F}$ and 2.7 V DC rating, can store $364 \mathrm{~J}$. Hence, supercapacitors have a clear volumetric (space) advantage compared to inductors.
In addition, commercial SCs have lower ESR (in the range of 0.1 to $100 \mathrm{~m} \Omega$ ) than electrolytic capacitors (30 to 1000 $\mathrm{m} \Omega$ ), making SCs less dissipative. Because of their small series resistance, the dissipative voltage loss across a seriesconnected SC can be close to negligible (e.g, 5 A through 1 $\mathrm{m} \Omega$ giving a $5 \mathrm{mV}$ potentially difference), while the potential difference $\Delta V$ across the capacitor can be large and only slowly varying as a constant current $I_{\mathrm{C}}$

$$
\Delta V=\frac{I_{\mathrm{C}} \Delta t}{C}
$$

since $\Delta V \sim 1 / C$. This means that the series-connected SC can be regarded as an essentially lossless voltage dropper. We can combine these desirable SC properties with loss minimization during charging (describe above) as the basis for two supercapacitor-assisted designs for DC regulation (SCALDO) and lighting (SCALED).

\section{A Typical New Application: The SCALDO TECHNIQUE}

SCALDO technique is one of the early applications of the concept discussed in the sections I and II. In this patented technique [15] the research team was able to combine a linear regulator with a supercapacitor or an array of supercapacitors to achieve a new converter topology with the following characteristics:

- Load receives the low-noise and high-current slew rate capable DC output of a linear regulator

- Switching frequency is extremely low

- RFI/ EMI issues are eliminated

- Output currents ranging from mA to over $100 \mathrm{~A}$

- DC-UPS capability

By using the SCALDO approach, ETTE of 5-3.3 V and $12-5 \mathrm{~V}$ converters can be improved by multiplication factors of 1.33 and 2 respectively. A linear 5-3.3 V converter with nominal $66 \%$ of ETEE can be theoretically increased to $99 \%$, and $12-5 \mathrm{~V}$ linear converter $42 \%$ efficiency can be increased to $84 \%$.

\section{A. SCALDO Basics}

Figure 3 depicts the basic SCALDO technique. In Figure 3, the low drop-out regulator works within an input range of 5.3 to $6 \mathrm{~V}$ maintaining an efficiency of $83 \%$. By using two series switches at "a" and "b" with an average capacitor voltage of $6 \mathrm{~V}$, the circuit will regulate until the capacitor reaches a maximum voltage of $6.7 \mathrm{~V}$ at which point the LDO reaches its lowest input voltage of $5.3 \mathrm{~V}$. A controller then switches the capacitor and the LDO configuration Figure 3(b) to allow the circuit to maintain overall charge balance of supercapacitor across cycles. In this case of 12 -V-to- $5 \mathrm{~V}$ regulator, we achieve a theoretical ETEE improvement factor of 2, since half of the time the input $12 \mathrm{~V}$ source is disconnected as the capacitor drives the LDO. More details on the SCALDO converter are in $[6,14,16,17]$. 


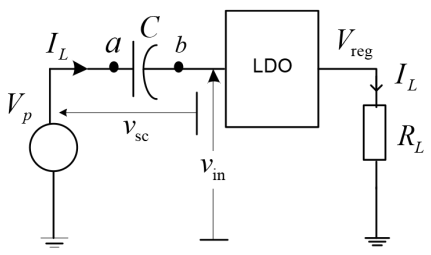

(a)

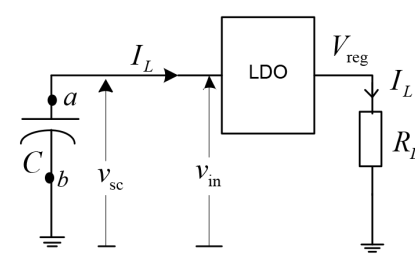

(b)
Fig. 3: The concept of supercapacitor energy storage and recovery (a) Minimizing the series element dissipation (b) Releasing stored energy [14]

\section{B. Switched Capacitor Converters (SWC) vs SCALDO}

During the early stages of SCALDO development, questions were raised if SCALDO is a variation of SWC converters. To provide a clear topological distinction, compare the voltage doubler SWC in Figure 4 with a SCALDO circuit.

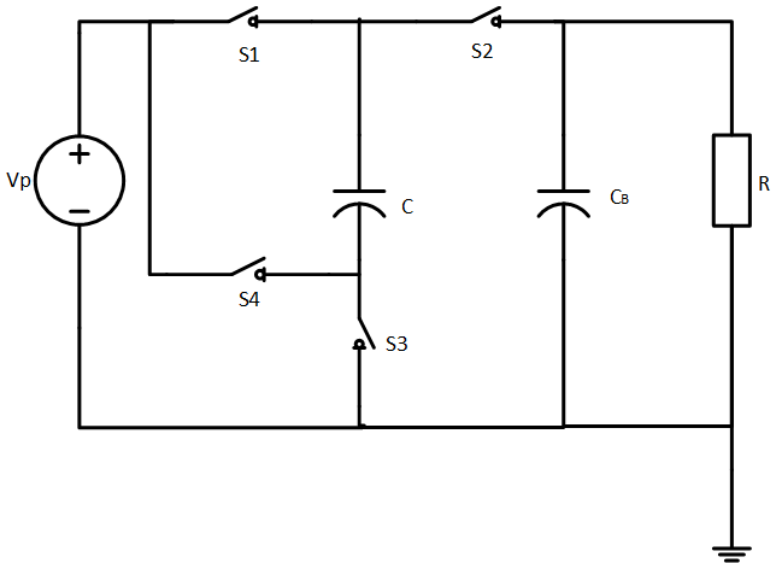

Fig. 4: Voltage doubler circuit in switched capacitor circuit

During the charging phase of Figure 4, S1 and S3 are closed while S2 and S4 are opened and the capacitor $C$ is connected in parallel with the power supply and charged up to $V_{\mathrm{P}}$. During this time capacitor $C_{\mathrm{B}}$ discharges to the load. During the discharging phase, $\mathrm{S} 2$ and $\mathrm{S} 4$ are closed and $\mathrm{S} 1$ and $\mathrm{S} 3$ are opened, placing the input source and the capacitors $C$ in series to give approximately twice the input voltage across the capacitor $C_{\mathrm{B}}$. In this phase, $C$ is connected series with the power supply.

Note that in this switched capacitor converter, the capacitor charges directly from unregulated power supply. In SCALDO converters, the capacitor is never placed in parallel with the input power supply, so SCALDO is not a topological variant of the classical switched capacitor converters.

Charge pumps are designed to operate at fixed switching frequency (few $100 \mathrm{kHz}$ ). Switched capacitor converters are limited to low to medium power level (several $10 \mathrm{~s}$ of $\mathrm{mW}$ ) applications due to practical limitations on switches and size of the capacitors.
The three significant features that distinguish SWC from SCALDO are as follows:

- A capacitor does not get directly charged from the input DC source during any phase of a SCALDO converter

- SCALDO runs at extra low frequencies such as millihertz to hertz

- In SCALDO, a very large capacitor is used in one operational phase as a semi-ideal lossless voltage dropper; this concept is never used in switched capacitor converters. A detailed comparison of these techniques is presented in [6].

\section{OTHER COMmercially Viable Supercapacitor Assisted (SCA) TECHNIQUES}

In this section we provide a summary of four SCA techniques.

\section{A. (SCALED) Technique for DC-Microgrids}

In a classical inverter-based renewable energy supply shown in Figure 5, the ETEE is decided by the product of individual efficiencies of each stage (DC-DC converter, inverter, 230-Vto-12-V LED driver). However, commercially available $12 \mathrm{~V}$ LED lamps can operate in a wide range of input voltages around the nominal voltage of $12 \mathrm{~V}$ [18]. Figure 6a indicates this property for commercial $12 \mathrm{~V}$ LED lamps tested in laboratory, maintaining a constant brightness, and Fig $6 \mathrm{~b}$ shows the approximately constant current region. This useful property could be combined with a supercapacitor bank in a DC micro-grid environment fed by renewable energy sources to achieve high ETEE, while SCs are used to buffer the temporary input-energy fluctuations.

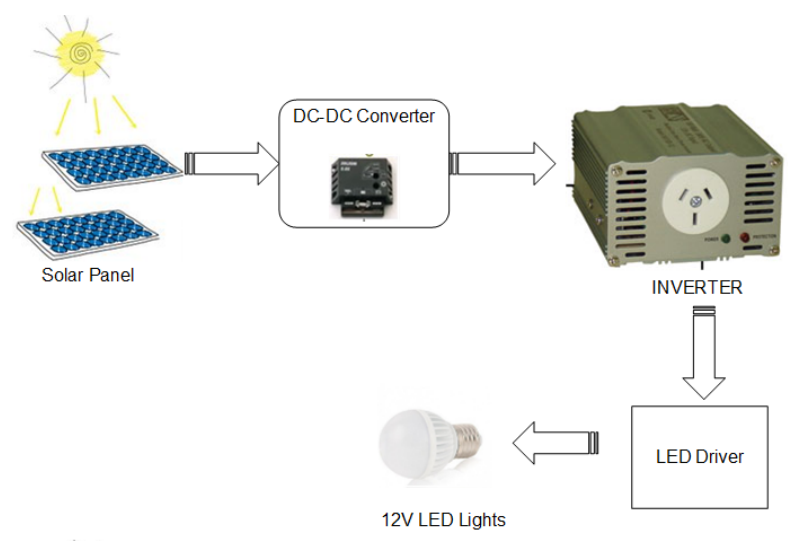

Fig. 5: Existing solar power based LED lighting system

By combining such a $12 \mathrm{~V}$ LED lamp and a SC bank, where SC bank gets charged from the renewable source as in Figure 7, we apply the theoretical concept of Figure 2(a). For reasons of commercial sensitivity, a detailed discussion on this very low frequency conversion technique is not presented in this paper. This project which is currently in progress aims at buffering low-voltage capable LED lamps by a seriesconnected SC bank to apply the loss-circumvention principle 


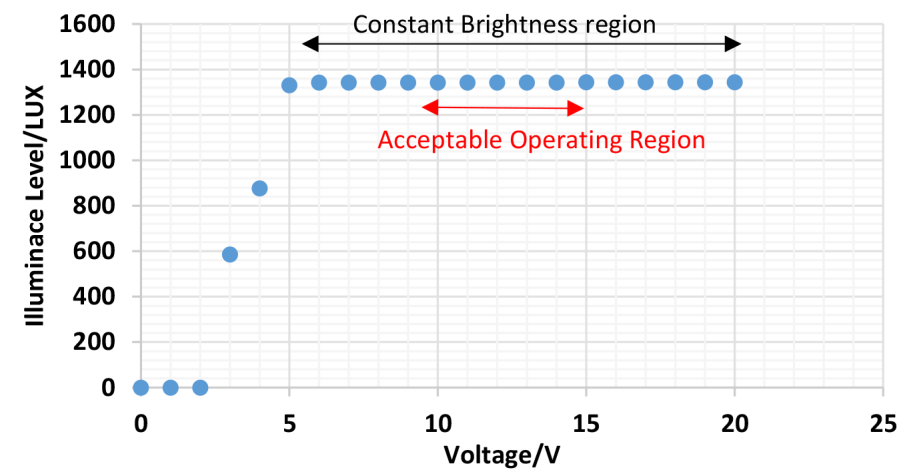

(a)

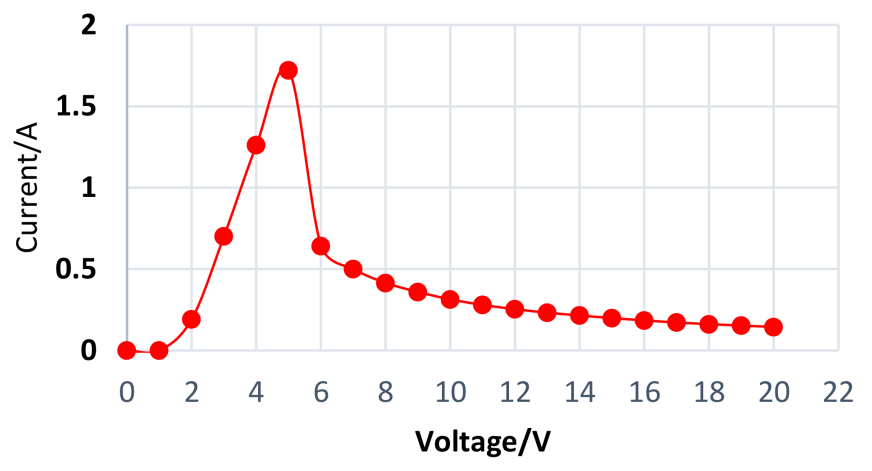

(b)

Fig. 6: Properties of commercial LED lamps (a) Variation of LUX level with LED voltage (b) Variation of LED current with voltage

explained earlier. Here, the LED lamp acts as the resistive load $R_{\mathrm{L}}$ in Figure 2(a).

The SCALED technique presently under development is expected to achieve the following:

- short term energy storage providing DC-UPS capability

- buffer unsafe voltages to power low voltage LED lamps

- higher ETEE

- minimizing RFI/ EMI issues

- tolerate wider DC - voltage fluctuations of the renewable source

\section{B. SC-Assisted Surge Absorber}

Supercapacitors are capable of delivering and receiving high currents. The surge absorbent capability used to develop the Supercapacitor-Assisted Surge Absorber (SCASA), is a patented and commercialized technique developed at the Power Electronics Research Group, University of Waikato. The component count of SCASA is lower than alternative commercial products giving a manufacturing cost advantage. SCASA topology can be used as a base technique to develop full-scale common and differential mode surge capable, fully versatile, commercial surge protectors with better performance than traditional surge protectors [19, 20].

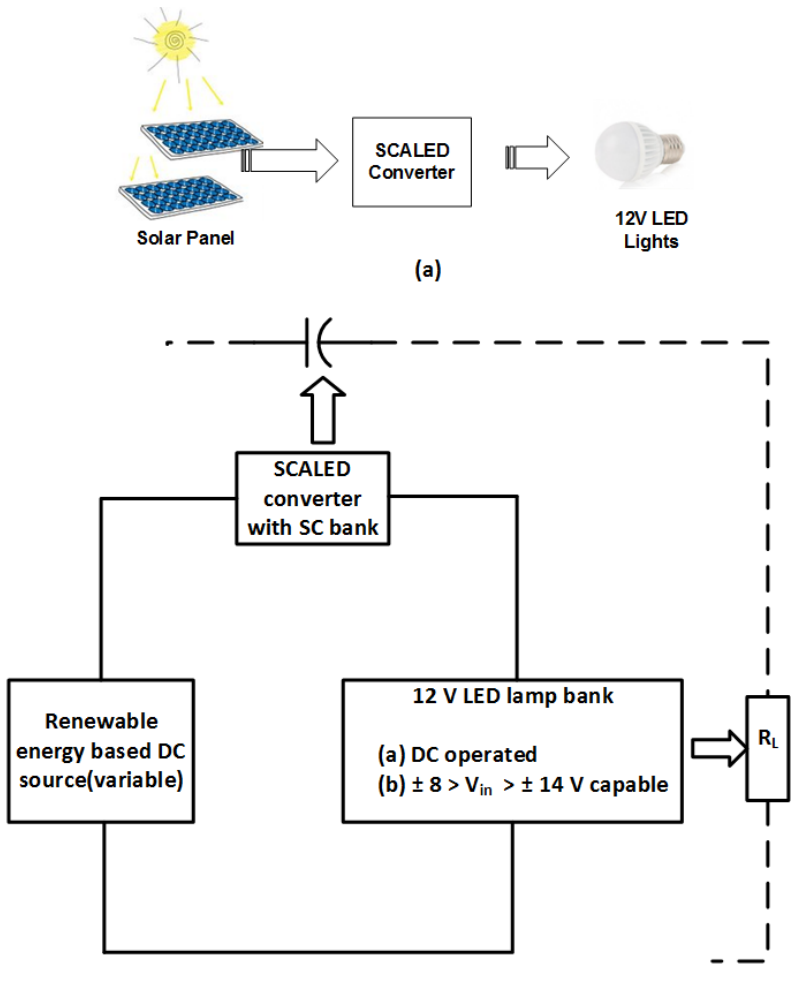

(b)

Fig. 7: Concept of SCALED (a) simplified block diagram (b) pictorial view

\section{SC-Assisted Temperature-Modification Apparatus}

SC-Assisted Temperature-Modification Apparatus (SCATMA) is an "instant water heating technique" based on prestored SC energy to overcome the delayed delivery of hot water in domestic water taps [21]. This technique utilizes a pre-charged low-voltage SC bank with short-term current delivery capability in the range of $600-2400$ A. The system uses SC cells in the range of 1200-5000 F with storage capability of $0.6-3 \mathrm{Wh}$. A comprehensive discussion on this low-voltage rapid-heat delivery technique is in [22].

\section{SC-Assisted High-Density Inverter}

SC-Assisted High-Density Inverter (SCAHDI) is an application where a resistive inverter load is placed in the capacitor charging loop to achieve a high-density inverter. The fundamental concept of SCADHI is to partition the overall inverter requirement into smaller inverters powered by a half of the nominal voltage of the DC source such as a solar supply. The second half of the inverter is fed by an input dc source in series with the discharged SC banks. The output voltage of the overall system is determined by the series-connected outputs of the smaller inverters $[23,24]$.

\section{CONCLUSION}

In this paper the RC charging loop has been analysed with the goal of optimizing capacitors-charging efficiency while inserting a useful resistive load in the loop to recover 
a substantial fraction of the wasted energy. Application of this loss circumvention principle in the RC charging circuit has allowed the power electronic research team at the University of Waikato to develop several novel, non-traditional supercapacitor-based circuit topologies such as SCALDO, SCASA, SCATMA, SCADHI and SCALED.

\section{REFERENCES}

[1] M. T. Zhang, M. M. Jovanovic, and F. C. Lee, "Design considerations for low-voltage on-board DC/DC modules for next generations of data processing circuits," IEEE Transactions on Power Electronics, vol. 11, no. 2, pp. 328-337, 1996.

[2] G. Bocock, "Power Supply Technical Guide 2010/11," tech. rep., XP Power, 2010.

[3] H. Guldner, F. Eckholz, H. Wolf, and J. Losansky, "A voltage regulator module (VRM) application for a switched mode power supply (SMPS)," in VIII IEEE International Power Electronics Congress, 2002. Technical Proceedings. CIEP 2002., pp. 139-144, Oct 2002.

[4] Y. F. Liu, E. Meyer, and X. Liu, "Recent Developments in Digital Control Strategies for DC/DC Switching Power Converters," IEEE Transactions on Power Electronics, vol. 24, pp. 2567-2577, Nov 2009.

[5] N. Kularatna, DC Power Supplies Power Management and Surge Protection for Power Electronic Systems. September 2012.

[6] K. Kankanmage and N. Kulatana, "Supercapacitor assisted LDO (SCALDO) techniquean extra low frequency design approach to high efficiency DC-DC converters and how it compares with the classical switched capacitor converters," in 2013 Twenty-Eighth Annual IEEE Applied Power Electronics Conference and Exposition (APEC), pp. 1979-1984, March 2013.

[7] J. F. Dickson, "On-chip high-voltage generation in MNOS integrated circuits using an improved voltage multiplier technique," IEEE Journal of Solid-State Circuits, vol. 11, pp. 374-378, Jun 1976.

[8] G. Palumbo and D. Pappalardo, "Charge Pump Circuits: An Overview on Design Strategies and Topologies," IEEE Circuits and Systems Magazine, vol. 10, pp. 31-45, First 2010.

[9] G. A. Rincon-Mora and P. E. Allen, “A low-voltage, low quiescent current, low drop-out regulator," IEEE Journal of Solid-State Circuits, vol. 33, pp. 36-44, Jan 1998.

[10] G. A. Rincon-Mora and P. E. Allen, "Optimized frequency-shaping circuit topologies for LDOs," IEEE Transactions on Circuits and Systems II: Analog and Digital Signal Processing, vol. 45, pp. 703-708, Jun 1998.

[11] A. B. da Cunha and D. C. d. Silva, "Energy-efficient characterization of solar panel-supercapacitors systems for energy-harvesting aware wireless sensor nodes," in 2009 IEEE 20th International Symposium on Personal, Indoor and Mobile Radio Communications, pp. 22752279, Sept 2009.
[12] Z. Yin, M. Han, Y. Du, and Z. Zhang, "A Practical Approach for Ride Through of Super Capacitor Energy Storage Based ASD System," in 2005/2006 IEEE/PES Transmission and Distribution Conference and Exhibition, pp. 744-746, May 2006.

[13] Y. Zhang and Z. Jiang, "Dynamic power sharing strategy for active hybrid energy storage systems," in 2009 IEEE Vehicle Power and Propulsion Conference, pp. 558-563, Sept 2009.

[14] K. Gunawardane, Analysis on Supercapacitor Assisted Low Dropout (SCALDO) Regulators. PhD thesis, University of Waikato, 2014.

[15] N. Kularatna and J. Fernando, "High current voltage regulator,' March 2011.

[16] N. Kularatna, "Supercapacitor Assisted Low Dropout Regulators (SCALDO) for high efficiency DC-DC converters for DC microgrid applications," in 2015 IEEE First International Conference on DC Microgrids (ICDCM), pp. 333-338, June 2015.

[17] T. Wickramasinghe, N. Kularatna, and D. A. Steyn-Ross, "Reduced-switch SCALDO technique for high-current VRM implementation," in IECON 2013 - 39th Annual Conference of the IEEE Industrial Electronics Society, pp. 6789-6793, Nov 2013.

[18] J. Fernando and N. Kularatna, "Supercapacitors for distributed energy storage in DC microgrids and loads," in 2015 IEEE First International Conference on DC Microgrids (ICDCM), pp. 339-342, June 2015.

[19] J. Fernando, N. Kularatna, H. Round, and S. Talele, "Implementation of the supercapacitor-assisted surge absorber (SCASA) technique in a practical surge protector," Oct 2014.

[20] J. Fernando and N. Kularatna, "Supercapacitor assisted surge absorber (SCASA) technique: Selection of supercapacitor and magnetic components," in 2014 IEEE Energy Conversion Congress and Exposition (ECCE), pp. 1992 1996, Sept 2014.

[21] N. Gurusinghe, N. Kularatna, W. H. Round, and D. A. Steyn-Ross, "Energy-Limited Transient-Mode Fast Supercapacitor Charger Topology," IEEE Transactions on Power Electronics, vol. 32, no. 2, pp. 911-914, 2017.

[22] N. Gurusinghe, Supercapacitor Assisted Temporature Modification Apparatus (SCATMA) and Fast Supercapacitor Charger. $\mathrm{PhD}$ thesis, University of Waikato, 2016.

[23] N. Kularatna, K. Milani, and W. H. Round, "Supercapacitor energy storage in solar application: A design approach to minimize a fundamental loss issue by partitioning the load and the storage device," in 2015 IEEE 24th International Symposium on Industrial Electronics (ISIE), pp. 1308-1312, June 2015.

[24] N. Kularatna, D. A. Steyn-Ross, and K. Milani, “A designer's view on non-traditional supercapacitor techniques for sustainable energy applications," in 2016 IEEE 25th International Symposium on Industrial Electronics (ISIE), pp. 962-967, June 2016. 